\section{Is rituximab effective for IgG4-related disease in the long term? Experience of cases treated with rituximab for 4 years}

A prospective open-label trial of rituximab (RTX) for IgG4-related disease (IgG4-RD) was recently described in the Annals of the Rheumatic Diseases by Carruthers et al. ${ }^{1}$ According to their results, RTX is effective as induction therapy for IgG4-RD without glucocorticoid in the short term. We agree and support their results. We have also prescribed RTX for typical cases of IgG4-RD, showing characteristics of younger age, experience of several relapses, no history of hepatitis B and, since 2011, hesitation to increase the dose of glucocorticoid due to complications. We are currently treating three cases using RTX. Our protocol is as follows. We prescribe $500 \mathrm{mg} /$ body of RTX at the onset of relapse. Meanwhile, the dose of glucocorticoid is decreased as much as possible. The patients visit our hospital for blood tests once every few months. Whole-body CT is routinely performed once a year. When relapse is suspected, we examine the whole body by CT.

The maximum period of follow-up since initial RTX is currently 4 years. Case 1 involved a 60 -year-old Japanese woman. She presented with IgG4-related dacryoadenitis and sialadenitis, and autoimmune pancreatitis. The initial dose of glucocorticoid was $40 \mathrm{mg} /$ day, but relapses repeated with the tapering of steroid doses. Immunosuppressants were added, but proved ineffective and a third relapse was experienced in 2011. RTX was started because of glucocorticoid-induced avascular necrosis of the femoral heads. The glucocorticoid dose is currently $4 \mathrm{mg} /$ day. Case 2 involved a 42-year-old Japanese woman who initially suffered from IgG4-related dacryoadenitis and sialadenitis. IgG4-related tubulointerstitial nephritis and retroperitoneal fibrosis occurred during the course. The initial dose of prednisolone was $30 \mathrm{mg} / \mathrm{day}$, but a third relapse was experienced in 2012. In this case, increasing the dose of glucocorticoid was avoided due to the complication of severe diabetes mellitus. RTX was started and the dose of prednisolone was decreased to $3 \mathrm{mg} /$ day. Case 3 involved a 44-year-old Japanese man. The patient showed lesions of the lacrimal and salivary glands, pancreas and lungs. The initial dose of steroid was $30 \mathrm{mg} /$ day. The patient experienced two relapses despite a combination of immunosuppressants, such as methotrexate and cyclophosphamide. We administered RTX in 2013 due to impaired glucose tolerance. The dose of prednisolone is currently $4 \mathrm{mg} /$ day (table 1). Adverse events were only seen in case 1, as herpes zoster, and the other patients showed no problems. ${ }^{2}$

Figure 1 shows serial changes in serum levels of IgG4 and the percentage of CD19+ lymphocytes among all lymphocytes after initial RTX prescription in these three cases. After the initial administration of RTX, five relapses were seen in case 1, two in case 2 and one in case 3. Levels of serum IgG4 after RTX therapy were elevated at relapse in case 1 . The percentage of CD19+ lymphocytes was also elevated at relapse. In case 2, serum IgG4 levels were not elevated at first relapse after RTX

Table 1 Profiles of patients with IgG4-related disease treated with RTX

\begin{tabular}{|c|c|c|c|c|c|c|c|c|c|c|}
\hline Case & Age, sex & 001 & Complications & $\begin{array}{l}\text { Initial } \\
\text { dose of } \\
\text { PSL }\end{array}$ & Immunosuppressants & $\begin{array}{l}\text { Times of } \\
\text { relapse }\end{array}$ & $\begin{array}{l}\text { Pre-treatment } \\
\text { of RTX }\end{array}$ & $\begin{array}{l}\text { Times } \\
\text { of RTX }\end{array}$ & $\begin{array}{l}\text { Period of } \\
\text { follow-up } \\
\text { (months) }\end{array}$ & $\begin{array}{l}\text { Treatment } \\
\text { at this point }\end{array}$ \\
\hline 1 & $60 \mathrm{~F}$ & AIP & G-AVN & 40 & CsA, MZR & 3 & PSL 16+MZR & 6 & 48 & PSL 4 \\
\hline 2 & $42 \mathrm{~F}$ & TIN, RF & DM & 30 & AZA & 3 & PSL 15+AZA & 3 & 35 & PSL 3 \\
\hline 3 & $44 \mathrm{M}$ & AIP, PI & & 30 & MTX, MZR, CY & 2 & PSL 14 & 1 & 24 & PSL 4 \\
\hline
\end{tabular}

AIP, autoimmune pancreatitis; AZA, azathioprine; CSA, cyclosporine A; CY, cyclophosphamide; DM, diabetes mellitus; G-AVN, glucocorticoid-induced avascular necrosis of femoral head; MTX, methotrexate; MZR, mizoribin; OOI, other organ involvements; PI, pulmonary involvement; PSL, prednisolone; RF, retroperitoneal fibrosis; RTX, rituximab; TIN, tubulointerstitial nephritis.

Figure 1 Serial changes in levels of serum IgG4 and percentage of CD19-positive lymphocytes at each visit after rituximab treatment.
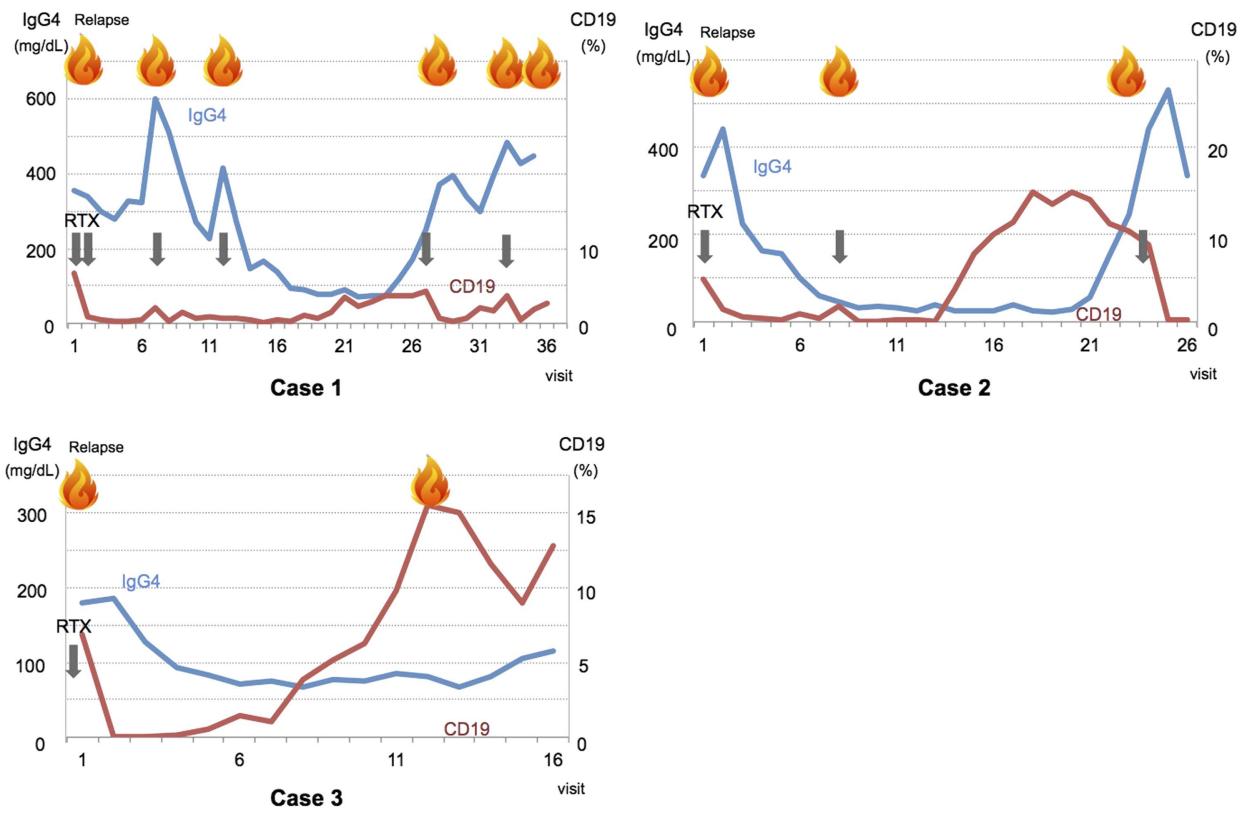
administration. On the other hand, CD19+ lymphocytes were increased, with a large fluctuation in range at both relapses. Case 3 did not present with elevated serum IgG4 concentration at relapse, but B cells were increased. With regard to case 1, which was treated multiple times with RTX, this treatment has recently tended to be less effective against IgG4-RD. The period of efficacy has become extremely short, instead of transitional. In this case, re-biopsy of lacrimal gland was performed, and development towards malignant lymphoma was ruled out. We performed further examination for anti-RTX antibody, but results were negative. The pathogenesis of case 1 has been shown to shifted towards RTX resistance.

The outcomes of our cases treated with RTX over the long term suggest two points. First, the indication for RTX to treat IgG4-RD is a definitive case ${ }^{3}$ diagnosed by histological examination, younger age, no history of hepatitis $\mathrm{B}$ and cases in which increasing the glucocorticoid dose is difficult due to complications. Second, one or two administrations of RTX cannot induce or maintain complete remission of IgG4-RD for long, and some cases present with attenuation of the RTX effect. The pathogenesis of IgG4-RD needs to be analysed and elucidated, ${ }^{4}$ and new treatment strategies need to be developed for RTX-resistant cases.

\section{Motohisa Yamamoto, ${ }^{1}$ Teruhito Awakawa, ${ }^{2}$ Hiroki Takahashi ${ }^{1}$}

${ }^{1}$ Division of Rheumatology and Clinical Immunology, Sapporo Medical University

School of Medicine, Sapporo, Japan

${ }^{2}$ Department of Internal Medicine and Gastroenterology, Sapporo Dohkohkai

Hospital, Sapporo, Japan

Correspondence to Dr Motohisa Yamamoto, Division of Rheumatology and Clinical Immunology, Sapporo Medical University School of Medicine, South

1-West 16, Chuo-ku, Sapporo, Hokkaido 0608543, Japan; mocha@cocoa.plala.or.jp

Acknowledgements We wish to thank Yui Shimizu, Hidetaka Yajima and Chisako Suzuki at Sapporo Medical University for the medical care of these cases.
Contributors MY, TA and HT contributed equally to the conception and drafting of the manuscript.

Competing interests None.

Ethics approval The Sapporo Medical University Hospital Institutional Review Board approved this study.

Patient consent Obtained.

Provenance and peer review Commissioned; internally peer reviewed.

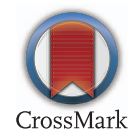

To cite Yamamoto M, Awakawa T, Takahashi H. Ann Rheum Dis 2015;74:e46.

Received 20 March 2015

Accepted 23 March 2015

Published Online First 10 April 2015

\section{(S) Linked}

http://dx.doi.org/10.1136/annrheumdis-2015-207640

Ann Rheum Dis 2015;74:e46. doi:10.1136/annrheumdis-2015-207625

\section{REFERENCES}

1 Carruthers MN, Topazian MD, Khosroshahi A, et al. Rituximab for IgG4-related disease: a prospective, open-label trial. Ann Rheum Dis 2015. Published Online First: 9 February 2015. doi:10.1136/annrheumdis-2014-206605

2 Yamamoto M, Yajima $\mathrm{H}$, Takahashi $\mathrm{H}$, et al. Everyday clinical practice in IgG4-related dacryoadenitis and/or sialadenitis: results from the SMART database. Mod Rheumatol 2015;25:199-204.

3 Umehara H, Okazaki K, Masaki Y, et al. Comprehensive diagnostic criteria for IgG4-related disease (IgG4-RD), 2011. Mod Rheumatol 2012;22:21-30.

4 Yamamoto M, Takahashi H, Shinomura Y. Mechanisms and assessment of IgG4-related disease: lessons for the rheumatologist. Nat Rev Rheumatol 2014;10:148-59. 